\title{
Photochemical Hydrogen Production with Metal-Organic Frameworks
}

\author{
Sonja Pullen ${ }^{1} \cdot$ Sascha $_{\text {Ott }}{ }^{1}$
}

Published online: 19 August 2016

(c) The Author(s) 2016. This article is published with open access at Springerlink.com

\begin{abstract}
Metal-Organic Frameworks (MOFs) have attracted increasing attention for the creation of solid-state platforms for catalysis applications. In this review article, we present strategies to employ MOF-based materials in photochemical hydrogen production. The scope ranges from the incorporation of single functions (catalyst or photosensitizer) to multifunctional MOFs that combine both light-harvesting and catalysis in one scaffold.
\end{abstract}

Keywords Metal-organic Framework · Light-harvesting · Photocatalysis · Hydrogen

\section{Introduction}

Hydrogen is a carbon neutral energy carrier that can be formed by reduction of water. As such it is considered to take a central role in future energy storage and supply $[1,2]$. The use of sunlight for the direct conversion of water into fuel is envisioned as essential process for sustainable production of hydrogen [3-5]. Metal-Organic Frameworks (MOFs) are a class of highly porous materials with exceptionally high surface area. Among many applications, they are being used for gas separation and storage [6, 7], chemical sensing [8], drug-delivery [9, 10] and various fields in catalysis [11-13]. Especially in the context of catalysis, MOFs continue to obtain increasing attention

Sonja Pullen

sonja.pullen@kemi.uu.se

$\square$ Sascha Ott

sascha.ott@kemi.uu.se

1 Department of Chemistry, Ångstrom Laboratories, Uppsala University, Box 523, 75120 Uppsala, Sweden since they offer a versatile solid-state platform for singlesite, heterogeneous, and stable catalysis. Many MOFs provide exceptionally high thermal and chemical stability, and can therefore provide a robust scaffold for the incorporation of potentially labile molecular catalysts. Also in the context of light-to-fuel conversion schemes, MOFs have emerged as support materials and some even take an active role in either sensitization or catalysis $[14,15]$. This review article summarizes different strategies on creating photocatalytic MOF-based schemes for the production of molecular hydrogen. Such schemes can be MOFs which have either catalysts or photosensitizers incorporated into their framework. More advanced examples carry both functions together, sometimes making use of both linker and metal-cluster of the MOF. Another route is the mixture of MOFs with solid photosensitizing units and catalysts to form composite materials active for photochemical hydrogen evolution.

\section{Suitable MOFs as Scaffold for Photocatalytic Hydrogen Production}

Metal-Organic frameworks are porous coordination polymers consisting of metal clusters that are inter-linked by organic molecules. Typically, the linkers contain two or more carboxylate-, pyridyl- or azolate functions that coordinate to the cluster. Using MOFs as scaffolds for catalysis applications is particularly appealing because they offer a great variability in design. A commonly used linker is terephthalic acid, which can carry additional functional groups such as an amino substituent. Such functional groups can easily be introduced during synthesis and be further functionalized [16, 17]. Also, the pore size can be specifically designed. For example by extending 
terephthalic acid to biphenyl-dicarboxylic acid, MOFs with similar topologies, but larger pore sizes are attainable. Table 1 summarizes the most important MOFs that have been used in context of photochemical hydrogen production and that are discussed in this paper. For a more general review on MOF structures, we recommend some excellent reviews [18-20].

A MOF platform used in hydrogen evolution schemes is required to be stable under catalytic conditions, in most cases in aqueous solution or aqueous/organic solvent mixtures at a range of different $\mathrm{pH}$. A detailed review on water stability of MOFs was published by Burtch et al. in 2014. [28] Among the most water-stable MOFs are UiO$66(\mathrm{Zr})(\mathrm{UiO}=$ University in Oslo) [21], MIL-53 (Al and $\mathrm{Cr})(\mathrm{MIL}=$ Materials Institute Lavoisier) [23], MIL-101 (Cr) [24] and MIL-125(Ti) [25] as well as the ZIF series (ZIF $=$ Zeolithic Imidazolium Frameworks) [27]. Another important water-stable MOF used for photochemical hydrogen production schemes in some examples that will later be discussed in this paper is UiO-67 ( $\mathrm{Zr}$ ) [29]. A main factor determining a MOF's thermodynamic stability in water is the strength of the metal-to-ligand bond, which depends on the basicity $\left(\mathrm{pK}_{\mathrm{a}}\right)$ of the linker and Lewisacidity of the metal [28]. In comparison to water $\left(\mathrm{pK}_{\mathrm{a}}\right.$ 15.7), e.g. imidazolium-based linkers with high $\mathrm{pK}_{\mathrm{a}}(18.6)$ combined with e.g. Zn clusters form extremely stable ZIFtype frameworks [30]. Another important characteristic that determines water stability is the connectivity of the metal cluster. For example, MOFs of the UiO-series contain $\mathrm{Zr}_{6}(\mathrm{OH})_{4} \mathrm{O}_{4}$-clusters, each connected to 12 linkers, that gives rise to extraordinary stability against hydrolytic decomposition [22], even though the carboxylic acid linkers possess a lower $\mathrm{pK}_{\mathrm{a}}$ than $\mathrm{H}_{2} \mathrm{O}$.

In our opinion, another very important factor for the choice of a MOF is the possibility of functionalization. As mentioned earlier, a large number of MOFs can be prepared in which functional linkers can already be introduced during synthesis of the framework [11]. These include mostly linkers with small organic functional groups, but there are also some examples where metal-complexes can be directly incorporated during the process of solvothermal synthesis as metallo-linkers [15]. Metal-nanoparticles [31] and molecular catalysts that would not withstand the harsh synthesis conditions of MOFs have to be installed postsynthetically. A large number of methods for post-synthetic modifications on parent MOFs that contain for example amino groups [17, 32] or open coordination sites [33-35] have been developed throughout recent years. Another way is to perform a post-synthetic ligand exchange (PSE) (also referred to as solvent-assisted linker exchange $=$ SALE) with a linker that matches the topology of the linkers in the parent framework, [36] and that carries the desired functionality. While post-synthetic modification methods have been explored for many different MOFs, including the aforementioned water-stable frameworks, post-synthetic ligand exchange is a rather new but powerful concept. Examples have been shown even for highly stable MOFs such as UiO-66, MIL-53 and ZIF-8 [37-39].

Photocatalytic schemes for hydrogen formation require at least three different components: a catalyst, a photosensitizer and an electron source which often is a sacrificial reagent. There are a number of different ways how these can be combined with the MOF scaffold; in any case, pore dimensions of the framework play an important role. Sacrificial reagents and/or photochemically produced reductants need to diffuse into the pores in order to meet their MOF-immobilized reaction partners. Thus, frameworks with larger pores can be advantageous in such cases. On the other hand, small pore windows may be desirable if for example the catalyst is only encapsulated inside the pores like a "ship-in-a-bottle". Both strategies will be considered throughout this review.

\section{MOF-Based Photocatalytic Systems-An Overview}

Given the reasonably large number of suitable MOFs that exhibit both water-stability and that allow linker functionalization, a variety of different combinations for photocatalytic hydrogen production schemes have been developed. We have divided the different schemes in four categories. The first one includes all studies where the catalyst is immobilized in the MOF, while the photosensitizer is outside (in solution). The second category uses photoactive MOFs in which either the linkers or the metalclusters are contributing to the light harvesting process and the catalyst is in the reaction solution. A third category combines both functionalities in the MOF scaffold. In all three cases, there are examples with both molecular and heterogeneous catalysts and photosensitizers. In category four we will summarize studies on composite materials in which the MOF is mixed together with other solid materials to create hetero-junctions active for photochemical hydrogen production.

\subsection{MOF-Catalysts with Photosensitizers in Solution}

In this chapter, we will summarize the work on MOFs with incorporated catalysts that were used together with external photosensitizers for visible-light promoted photochemical hydrogen production, as schematically illustrated in Fig. 1. MOF-catalysts may range from frameworks loaded with catalytically active nanoparticles to molecular catalysts immobilized as linker molecules. Most common among the heterogeneous catalysts are Pt-nanoparticles. 
Table 1 Structures of most important MOFs used for photocatalytic hydrogen formation

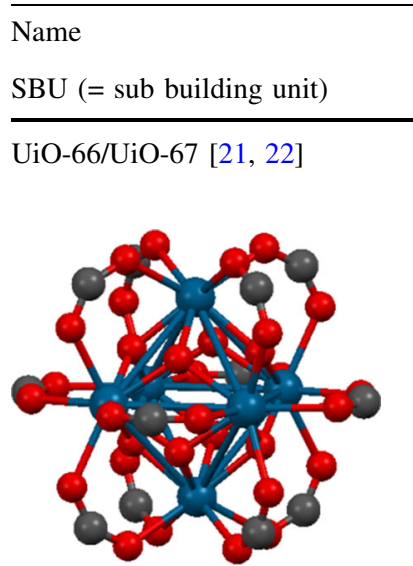

MIL-53 [23]

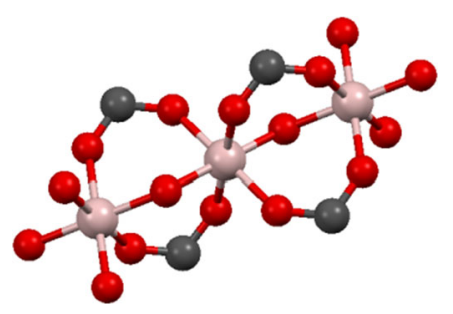

MIL-101 [24]

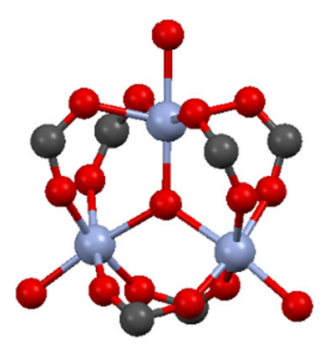

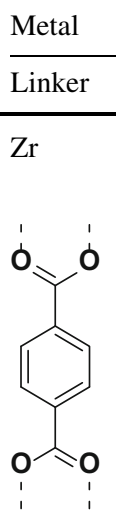

(N)<smiles>COC(=O)c1ccc(-c2ccc(C(=O)O)cc2)cc1</smiles>

$\mathrm{Al}$<smiles>COC(=O)c1ccc(C(=O)OC)cc1</smiles>

$\mathrm{Cr}$<smiles>COC(=O)c1ccc(C(=O)OC)cc1</smiles>

\section{Pore dimensions}

Structure

pore size

$6 \AA$ Å (UiO-66), $8 \AA$ (UiO-67)

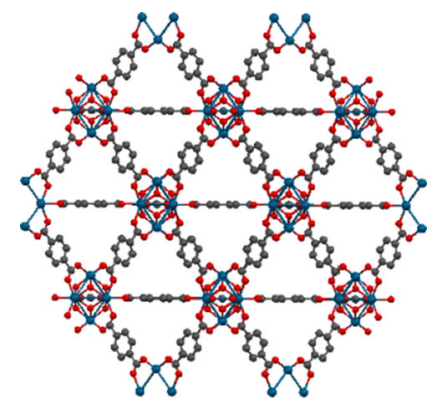

pore size: $8.5 \AA$

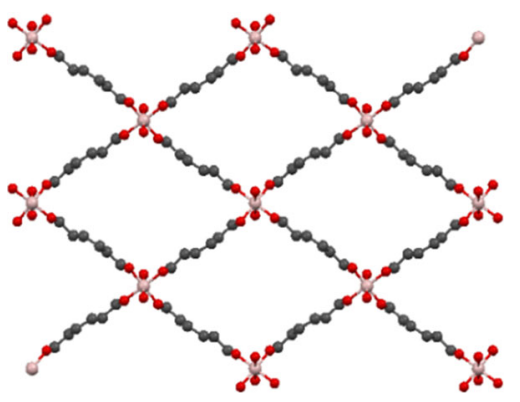

pore size: $29-34 \AA$,

(window 12-14.5 $\AA$ )
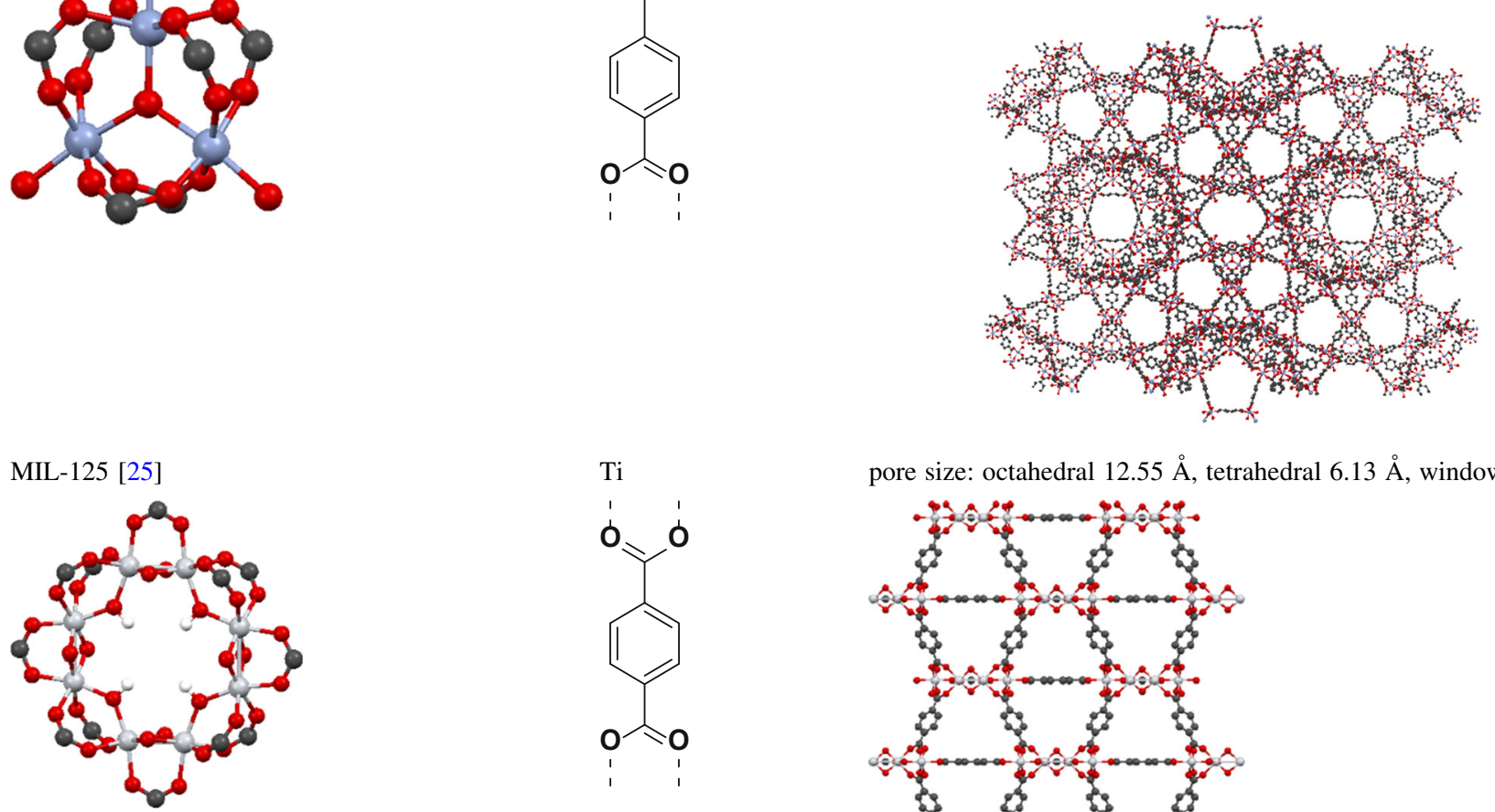

Ti

i $\rightleftharpoons$ o<smiles>COC(=O)c1ccc(C(=O)O)cc1</smiles>

pore size: octahedral $12.55 \AA$, tetrahedral $6.13 \AA$, window 5-7

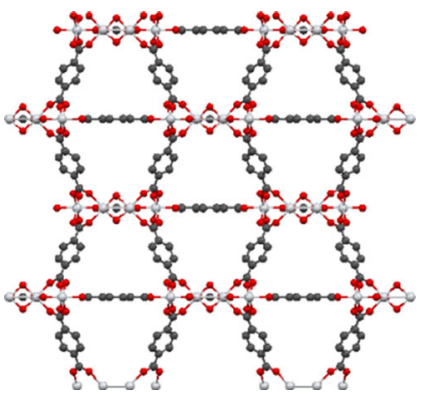


Table 1 continued

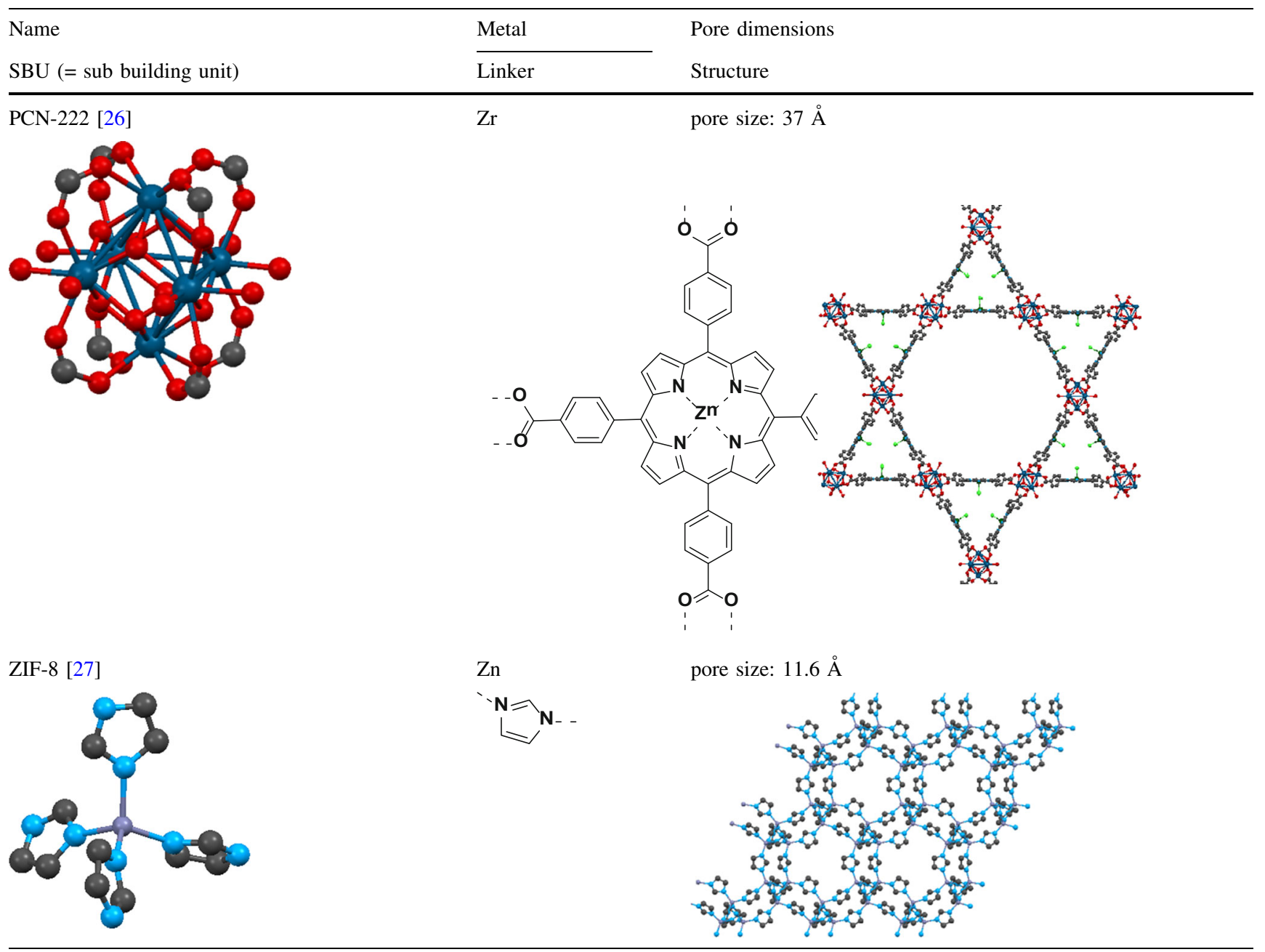

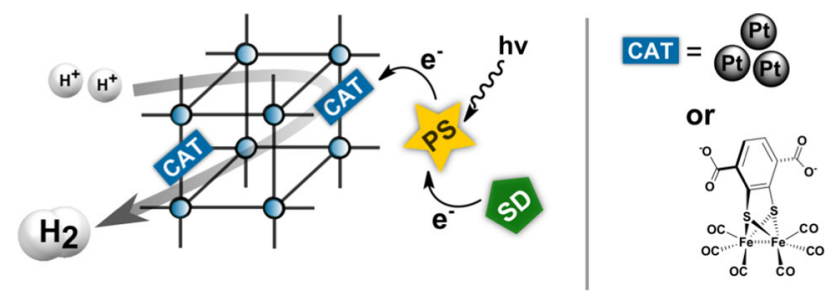

Fig. 1 Photocatalytic scheme for MOF-catalysts with external photosensitizer (PS). Sacrificial donor (SD) quenches the photosensitizer (PS), which reduces the catalyst (CAT) sitting inside the MOF. Catalysts used are Pt-nanoparticles [40-42] or the molecular diiron complex $[\mathrm{FeFe}](\mathrm{dcbdt})(\mathrm{CO})_{6}[43]$

In 2014, Yamashita and coworkers [41] reported an amine-functionalized MIL-101(Cr) loaded with Ptnanoparticles. As mentioned earlier, MIL-101(Cr) belongs to the most stable MOFs, even in acidic aqueous solution. The authors were able to vary the loading of Pt-nanoparticles onto the framework between 0.5 and $3.0 \mathrm{wt} \%$ by first soaking the $\mathrm{MOF}$ with $\mathrm{H}_{2} \mathrm{PtCl}_{4}$ and subsequent reduction with hydrogen. Crystallinity of the framework and homogeneity of particle size of the MOF were retained, while the BET surface area decreased by about $22 \%$ due to formation of Pt-nanoparticles inside the cavities. Transmission-electron microscopy (TEM) showed no significant agglomeration of nanoparticles. The photocatalytic activity was tested employing Rhodamine B as photosensitizer in an aqueous mixture with $20 \%$ triethanolamine (TEOA) as sacrificial electron donor. $\mathrm{NH}_{2}$-MIL-101(Cr) with Rhodamine $\mathrm{B}$ alone did also produce hydrogen, but the use of Pt-nanoparticles as co-catalyst resulted in the highest hydrogen production rate and turnover numbers. The optimal loading of Pt into the framework was shown to be $1.5 \mathrm{wt} \%$. Up to five cycles with slight decrease in activity were performed demonstrating the recyclability of their system.

In a similar fashion $\mathrm{He}$ et al. [40] employed a UiO66(Zr) framework as a platform to immobilize $\mathrm{Pt}$ nanoparticles as hydrogen evolution catalyst. In conjunction with Rhodamine B as photosensitizer, photocatalytic hydrogen formation under visible light irradiation in 
aqueous solution $\mathrm{pH} 7$ containing $10 \%$ TEOA as sacrificial donor could be demonstrated. In this study, the effect of varying concentrations of Rhodamine B was tested. In some experiments, the MOF was soaked with the dye prior to photocatalysis, in others it was added to the reaction solution. Questions remained whether the photoreaction is driven by rhodamine $\mathrm{B}$ that is in the interior of the MOF or in solution.

Yuan et al. [42] used Erythrosin B dye as sensitizer together with Pt-nanoparticles absorbed onto the surface of UiO-66(Zr). About $0.5 \mathrm{wt} \% \mathrm{Pt}$ was deposited on the MOF in situ by irradiating a suspension of UiO-66 in $\mathrm{H}_{2} \mathrm{PtCl}_{4}$ aqueous solution with 20 vol \% methanol as sacrificial electron donor. This Pt@UiO-66 catalyst was employed as active catalyst in an aqueous solution of the dye Erythrosin $\mathrm{B}$ and $\mathrm{L}$-ascorbic acid as sacrificial reagent at $\mathrm{pH} 4$. Again, concentration of the photosensitizer showed to be important. Addition of up to $30 \mathrm{mg}$ dye $(\sim 3.4 \mathrm{mM})$ to the reaction solution yielded in increasing hydrogen production, while more dye molecules could not further improve the performance. The authors did not specify if the system becomes limited by the catalyst at this point, which may be the case.

Liu et al. used MIL-101(Cr) for immobilizing Ni/NiOx nanoparticles as photocatalysts together with Erythrosin B as dye [44]. The nanoparticles were introduced by in situ photo-deposition in a suspension of the parent MOF in a solution of nickel nitrate, TEOA as sacrificial electron source and Erythrosin B dye as photosensitizer. This mixture showed to be active for hydrogen generation, while in absence of nickel nitrate no hydrogen was observed. The formation of $\mathrm{Ni}$ nanoparticles was observed by TEM. Variation of dye concentration did show similar results as the previous report; the additions of $30 \mathrm{mg}$ dye did lead to best performance, while even higher concentrations did not lead to further improvements. These results point towards limitations in the light-harvesting and photo-production of reducing equivalents.

The studies that are summarized above demonstrated that MOFs can serve as platforms for immobilizing nanoparticles that are known to be efficient catalysts for hydrogen formation. Tuning the systems with varying concentrations of dye molecules to harvest light efficiently often led to increased hydrogen production. The role of the MOF in such schemes is to provide a solid support with a high surface area which allows high catalyst density. Another branch in MOF research is the immobilization of molecular proton reduction catalysts. This strategy is mainly driven for two reasons: first, the integration of molecular catalysts in a solid support leads to easier separation and recyclability. The second idea is that the MOF scaffold potentially stabilizes labile catalytic intermediates by preventing charge recombination and thereby increasing the lifetime of the active species.

We recently reported the incorporation of a dinuclear iron complex into the robust and water-stable $\mathrm{UiO}-66(\mathrm{Zr})$ framework [43]. The complex $[\mathrm{FeFe}](\mathrm{dcbdt})(\mathrm{CO})_{6}$ (dcbdt $=1$,4-dicarboxylbenzene-2,3-dithiolate) is a functional mimic of the $[\mathrm{FeFe}]$-hydrogenase active site and serves as proton reduction catalyst. Post-synthetic ligand exchange facilitated the incorporation of the complex with matching ligand topology as the linker 1,4-benzene-dicarboxylic acid. About $14 \%$ of all linkers were exchanged by the catalyst as indicated by [1] H-NMR and energy dispersive X-ray (EDX), and the molecular integrity of the catalyst was proven by FT-IR and Extended X-ray absorption fine structure spectroscopy (EXAFS). In a photocatalytic scheme in aqueous suspension with $\mathrm{Ru}(\mathrm{bpy})_{3} \mathrm{Cl}_{2}$ (bpy $=2,2^{\prime}$-bipyridine) as photosensitizer and ascorbate as sacrificial donor at $\mathrm{pH} 5$, the MOF showed to be active as proton reduction catalyst. It even showed to be superior to the homogeneous system with the molecular catalyst under otherwise identical conditions. While the molecular catalyst rapidly decomposed under irradiation, the MOF still showed IR bands corresponding to the CO ligand of intact catalyst after several hours under photocatalytic conditions. This finding clearly shows that the conclusion of molecular catalysts in a MOF prolongs the lifetime of the catalyst.

Other molecular proton reduction catalysts have been incorporated into MOFs and some of the examples will be discussed later in this article, as they were integrated in MOFs with dual functionality. There are also a few MOFs which contain $\mathrm{CO}_{2}$ reduction catalysts such as $\mathrm{Mn}$ (dcbpy) $(\mathrm{CO})_{3} \mathrm{Br}$ [45] (bpydc $=2,2^{\prime}$-bipyridine-5, $5^{\prime}$ dicarboxylic acid $)$ and $\mathrm{Cp} * \mathrm{Rh} \quad\left(\mathrm{Cp}^{*}=\right.$ pentamethylcyclopentadiene) [46]. These will not be discussed in detail, but it is worth mentioning that these authors used postsynthetic metallation and post-synthetic exchange as incorporation methods. For both systems, the formation of hydrogen as side product was observed.

\subsection{MOF-photosensitizer schemes with external catalyst}

The second strategy is to incorporate photosensitizing units into the MOF scaffold and to use external catalysts to complete the system. Figure 2 illustrates a schematic overview.

In 2012, Fateeva et al. [47] presented a porphyrinebased MOF with aluminium nodes. The structure of this Al-MOF is comparable to MIL-60 [48]. The porphyrinecontaining Al-MOF showed to be water-stable in a $\mathrm{pH}$ range of 5-8 and $90 \%$ of the porphyrine sites could post- 


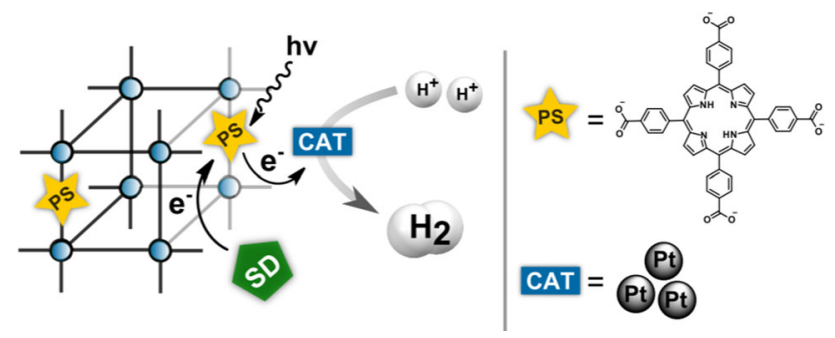

Fig. 2 Schematic photochemical hydrogen production with immobilized photosensizer (PS) and external catalyst (CAT). Sacrificial donors (SD) are typically ethylenediaminetetraacetic acid (EDTA) or triethylamine (TEA). In this case, the photosensitizer are porphyrine linkers and Pt-nanoparticles serve as catalyst [47]

synthetically be metallated with $\mathrm{Zn}^{+}$. Both, the nonmetallated and the metallated MOF were investigated in two different schemes for photocatalytic hydrogen formation under visibile light irradiation. The first scheme included Pt-nanoparticles as active catalyst, ethylenediamine tetraacetate (EDTA) as sacrificial electron donor and methylviologen as electron relay. Both MOFs showed hydrogen formation, however with a low quantum yield. The authors attributed the low activity to diffusion limitations of methylviologen. In a second scheme, methylviologen was omitted from the reaction mixture to afford direct electron transfer between the reduced MOF and colloidal platinum. At the same time, the concentration of Pt-catalyst was increased to ensure optimum contact. This system showed increased hydrogen production for both MOFs, while the metallated performed best. PXRD and SEM showed retained crystallinity of the MOFs after photocatalysis for $3 \mathrm{~h}$ and a control experiment with the supernatant of MOFs stirred in the reaction mixture in the dark for $24 \mathrm{~h}$ did proof that there was no leakage of porphyrine that would lead to homogeneous catalytic activity.(Fig. 3)

Lin and coworkers prepared two $\mathrm{UiO}(\mathrm{Zr})$ type MOFs doped with iridium photosensitizers, $\left[\operatorname{Ir}(\mathrm{ppy})_{2}(\mathrm{bpy}) \mathrm{Cl}\right]$ (ppy $=2$-phenyl-pyridine) and $\left[\operatorname{Ir}(\text { ppy })_{2}(\right.$ bpydb $\left.) \mathrm{Cl}\right]$ (bpy$\mathrm{db}=\left(2,2^{\prime}\right.$-bipyridine $)-5,5^{\prime}$-dibenzoate $)$ in a solvothermal synthesis process. Together with Pt-nanoparticles that were photo-deposited onto the frameworks, both MOFs were active for photocatalytic hydrogen formation under visible

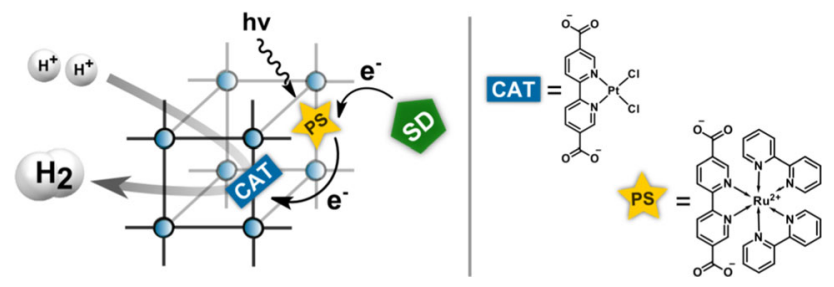

Fig. 3 MOF with dual functionality. In the depicted example by Hou and coworkers [49], two molecular species were co-incorporated into the MOF light irradiation with triethylamine (TEA) as sacrificial donor. Homogeneous control experiments revealed that the MOF with shorter linker had similar reactivity, while the larger linker could greatly enhance photocatalytic hydrogen formation.

\subsection{MOFs Containing Both Photosensitizer and Catalyst}

The MOF community has even developed scaffolds with dual functionality, e.g. frameworks containing both catalyst and photosensitizing units. These efforts demonstrate the power of MOFs as photocatalytic materials. Dual functionality could either be achieved by incorporating molecular catalysts and photosensitizers, or by a system in which one component fulfills both functions. A third strategy is the use of photoactive frameworks together with a catalyst.

The first example of this strategy was reported in 2013 by Zhou et al. [50] The authors presented a MOF containing a dichloro-2,2'-bipyridyl-platinum complex serving both as photosensitizer and catalyst. As platform they used MOF-253, which has the structure $\mathrm{Al}(\mathrm{OH})(\mathrm{dcbpy})$ and is known to have a high chemical and thermal stability as well as a rigid framework structure. Via post-synthetic metallation of the open bipyridine sites with cis$\mathrm{Pt}(\mathrm{DMSO}) \mathrm{Cl}_{2}$, a material with a $\mathrm{Pt} / \mathrm{Al}$ ratio of 0.5 was obtained. Photochemical hydrogen evolution was tested in an aqueous suspension at $\mathrm{pH} 8.5$ with 15 vol \% TEOA as sacrificial electron donor under visible light irradiation $(\lambda>420 \mathrm{~nm})$. Indeed, the material showed hydrogen formation up to $30 \mathrm{~h}$. A variety of control experiments including the use of the molecular complex cis$\mathrm{Pt}(\mathrm{DMSO}) \mathrm{Cl}_{2}$, Pt-nanoparticles or just the blank MOF-253 did not show any hydrogen formation. Additionally, the formation of Pt-nanoparticles during photocatalysis could be ruled out by a simple mercury test. EXAFS revealed a relatively short Pt-Pt distance of $3.6 \AA$, which seems to be essential for the proposed bimolecular intermediate in the photocatalytic cycle for hydrogen formation.

Employing a different framework (UiO-67(Zr)) but using the same catalyst, Hou et al. [49] reported the incorporation of $\mathrm{Pt}(\mathrm{dcbpy}) \mathrm{Cl}_{2}$ together with a photosensitizer $\mathrm{Ru}(\mathrm{dcbpy})(\mathrm{bpy})_{2} \mathrm{Cl}_{2}$ into a MOF. Both molecules were introduced during the solvothermal synthesis in a mix-and-match approach taking advantage of the matching linker topology of dcbpy and 4,4'biphenyl-dicarboxylic acid of the parent framework. A typical sample contained doping concentrations of $0.86 \% \mathrm{Ru}$ and $5.4 \% \mathrm{Pt}$. The samples were highly crystalline as indicated by PXRD and showed high porosity. Photocatalytic hydrogen evolution experiments were carried out in $0.1 \mathrm{M}$ pH 5 acetate buffer with EDTA-2Na as sacrificial donor under visible light 
irradiation. In comparison with the homogeneous control, the dual functionalized MOF showed enhanced hydrogen formation. Similar to the previous report, the authors proposed the close Pt-Pt distance in the framework as compared to solution to be the main reason for increased efficiency. But also the co-incorporation of the photosensitizer showed to be beneficial as the control with homogeneous sensitizer and Pt@UiO-67 did perform not as well as the dual-incorporated MOF system. In order to investigate the mechanism in more detail, the authors attempted to use transient absorption spectroscopy. These were, however, not successful and the proposed mechanism was supported by theoretical calculations and experiments on the homogeneous complexes instead.

Moving towards more abundant elements, Feng and coworkers [51] reported a bimolecular system with a porphyrine-based MOF (Zr-PF-MOF) holding a molecular mimic of the iron-hydrogenase active site coordinated to the $\mathrm{Zn}$-atom in the porphyrine. Combining the photosensitizing porphyrine linker units with a axially coordinated proton reduction catalyst led to a photoactive composite material. The Zr-PF-MOF precursor possesses exceptional stability, and can be exposed for $24 \mathrm{~h}$ to visible light irradiation in aqueous acetate buffer $(\mathrm{pH} 5)$, as evidenced by PXRD. Coordination of the molecular catalyst was achieved by soaking the MOF in a $0.1 \mathrm{M}$ solution of the complex for 2 days. Successful incorporation was demonstrated by FT-IR as well as fluorescence emission quenching of the Zn-porphyrine after soaking with the complex, indicating coordination of the catalyst. EDX and ICP-MS were used to determine that $25 \%$ of all porphyrines were decorated with a catalyst. Photocatalytic activity was tested in aqueous acetate buffer at $\mathrm{pH} 5$, with ascorbate as sacrificial electron donor. The bi-functional MOF-system showed increased stability and overall reactivity as compared to the homogeneous system.

In 2015, Zhang et al. [52] presented the use of a polyoxometallate (POM) catalyst embedded into the zirconiumbased UiO-67 containing $\mathrm{Ru}(\mathrm{dcbpy})(\mathrm{bpy})_{2} \mathrm{Cl}_{2}$ linkers as photosensitizing units. The authors made use of the highly cationic framework to encapsulate an anionic Well-Dawson type POM of the structure $\left[\mathrm{P}_{2} \mathrm{~W}_{18} \mathrm{O}_{62}\right]^{6-}$ during the solvothermal synthesis of the MOF. The POM concentration could be varied by adding different amounts of POM to the synthesis solution (final W/Zr ratio 0.24-3.40). Photocatalytic hydrogen formation under visible light irradiation was performed in $\mathrm{DMF} / \mathrm{CH}_{3} \mathrm{CN}$ with TEOA as sacrificial donor. Recovered POM@MOF after photocatalytic reaction showed only slight loss of activity and crystallinity within three cycles. However, leaching of $5.6 \%$ of the $\mathrm{Ru}$ photosensitizer into the solution was observed. Better performance of the system was observed by changing the conditions to DMF/acetonitrile solution with triethanolamine as sacrificial donor. Homogenous control experiments using the POM together with either $\mathrm{Ru}(\mathrm{bpy})_{3}$ or $\mathrm{Ru}(\mathrm{dmbpy})(\mathrm{bpy})_{2}$ (dmbpy $=5,5^{\prime}$-dimethyl$2,2^{\prime}$-bipyridine) did not show any hydrogen formation. The authors observed precipitate formation in both cases and attributed this to decomposition of the photosensitizer. An alternative explanation for the drastically different performance of POM@MOF versus the homogeneous systems was offered on the basis of the catalytic mechanism of the POM: cyclic voltammetry studies indicated that at least six or more electrons need to be injected to the POM to reduce protons. Therefore, encapsulating the POM into the photosensitizing MOF seemed striking as the proximity to several sensitizers probably leads to faster and more efficient electron transfer.

Finally, MOFs with dual functionality have been developed based on photosensitizing frameworks together with catalysts as depicted in Fig. 4. The starting point for this approach was a report in 2010 by Garcia and coworkers, who used $\mathrm{UiO}-66(\mathrm{Zr})-\mathrm{NH}_{2}$ for photochemical hydrogen evolution under UV-light in the presence of methanol [53]. They showed that the organic linker can be excited by UV irradiation, leading to a ligand to cluster electron transfer.

Two years later, Matsuoka and coworkers picked up on this idea and reported a Ti-based MOF that contains aminoterephthalic acid linkers with a structure similar to that of MIL-125(Ti) [54]. They reasoned that the more positive conduction band of the titanium-oxo cluster compared to zirconium could be favorable for driving visible light photocatalysis. Indeed, the MOF could be employed for hydrogen evolution together with photo-deposited Ptnanoparticles using TEOA in aqueous solution as sacrificial donor under visible light irradiation $(\lambda>420 \mathrm{~nm})$. The MOF showed slight degradation and slight decrease in surface area after $9 \mathrm{~h}$ of irradiation. A control experiment with simple terephthalic acid linkers in the framework did not show photocatalytic activity, demonstrating the importance of amino-functionalization for the performance. Also, the use of TEOA as sacrificial reagent showed to be important as no hydrogen formation was

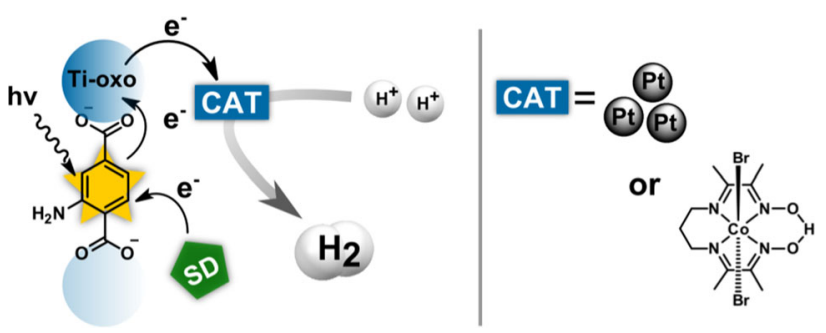

Fig. 4 Photochemical hydrogen production making use of photosensitizing framework. Pt-nanoparticles [54] or cobaloxime [55] were used as catalysts 
observed with TEA, EDTA or methanol. The authors attributed the need of TEOA to the weak oxidation power of the organic linker.

In 2015, Gascon and coworkers employed the same framework $\mathrm{NH}_{2}-\mathrm{MIL}-125(\mathrm{Ti})$ as photosensitizing platform for a molecular Co-oxime-diimine catalyst as guest molecule inside the framework's pores [55]. The molecular catalyst was build up inside the pores by first soaking the MOF with the oxime ligand and subsequent addition of $\mathrm{CoBr}_{2}$ leading to the formation of the catalyst. Taking advantage of the smaller size of the pore windows, the catalyst stays inside the MOF like a "ship-in-a-bottle". The authors employed a number of techniques including photoluminescence and EPR-spectroscopy to investigate the nature of the Co-MOF composite. However, they could not find conclusive evidence for the exact configuration of the catalyst inside the pores. In a photocatalytic scheme in acetonitrile with TEA as sacrificial reagent and trace amounts of water, hydrogen could be produced under visible light irradiation. The material achieved a 20-fold enhancement in hydrogen production compared to the photoactive framework without catalyst. In a control experiment with $\mathrm{D}_{2} \mathrm{O}$, they could show that water is indeed the proton source for their system. The catalyst could be recycled and used again for at least three cycles without significant loss of performance.

\subsection{MOF-Composites}

The final approach for making photo-active materials with MOFs is the combination with solid photosensitizers and catalysts to form a hetero-junction. In such composite materials the MOF does not take an active role in the photocatalytic process; it is rather used as a template to organize the other solid components.

$\mathrm{Wu}$ and co-workers prepared a UiO-66/CdS/graphene oxide composite [56]. Cadmium sulfide (CdS) was added to the framework by photodeposition of $\mathrm{S}_{8}$ and $\mathrm{CdCl}_{2}$ in ethanol solution. During this process, the structure of UiO66 was retained as indicated by PXRD. This material was employed together with reduced graphene oxide (RGO) in a photocatalytic scheme in aqueous solution containing $0.1 \mathrm{M} \mathrm{Na}_{2} \mathrm{~S}$ and $0.1 \mathrm{M} \mathrm{Na}_{2} \mathrm{SO}_{3}$ as well as Pt-nanoparticles $(0.5 \mathrm{wt} \%)$ as catalyst. In this scheme, CdS showed to be responsible for photo-generation of reducing equivalents as UiO-66/RGO alone did not produce any hydrogen. Improved activity as compared to commercial $\mathrm{CdS}$ particles was attributed to the large surface area of UiO-66 which could provide more catalytic active sites. RGO contributed to better conductivity.

He et al. [57] reported CdS embedded on MIL-101(Cr). Pt-nanoparticles were photo-deposited and found to build up on the surface of the CdS particles, as TEM images indicated. The system was tested for photocatalytic hydrogen formation in aqueous lactic acid solution under visible light irradiation. The MOF alone did not produce hydrogen, while sensitizing it with $\mathrm{CdS}$ and $0.5 \mathrm{wt} \% \mathrm{Pt}$ led to an active composite material.

Also UiO-66(Zr) in conjunction with $\mathrm{CdS}$ and $0.5 \mathrm{wt} \%$ Pt-nanoparticles showed to be an active catalyst, as reported by Zhou et al. [58]. CdS was deposited onto the MOF by in situ growth, and highest hydrogen production yields were achieved when $16 \mathrm{wt} \% \mathrm{CdS}$ was grown on the framework.

Finally, Yuan and coworkers [59] presented a UiO66/carbon nitride composite for visible light photochemical hydrogen formation. Both materials alone exhibit limited efficiency for hydrogen production, but the authors discovered that a junction of them could lead to enhanced activity. Carbon nitride $\left(\mathrm{g}-\mathrm{C}_{3} \mathrm{~N}_{4}\right)$ was deposited onto the surface of the MOF particles by annealing and PXRD showed that this procedure did not change the structure of the MOF. The authors could further show by TEM that the two materials had formed a clear interface between each other. Photochemical hydrogen evolution was tested in aqueous ascorbic acid solution $(\mathrm{pH} 4)$ under visible light irradiation. Again, $0.5 \mathrm{wt} \%$ Pt-nanoparticles was loaded in situ onto the composite as catalyst to drive the reaction. The best efficiency could be achieved with $50 \mathrm{wt} \%$ loading of carbon nitride.

\section{Summary and Conclusion}

In this review, we have summarized recent developments in the use of metal-organic frameworks for photochemical hydrogen production. Most commonly used MOF platforms are the UiO-66(Zr) and MIL-101(Cr) frameworks which are known for their high stability in aqueous media, as well as the $\mathrm{NH}_{2}$-MIL-125(Ti) framework which gained increasing interest recently due to light-induced linker-tocluster electron transfer properties that enable the light-tofuel conversion process. The role of MOFs in photochemical hydrogen evolution schemes ranges from simply providing a scaffold for the incorporation of light harvesting units and catalysts to the active involvement of metal-clusters in the photocatalysis process. In all areas of development, Pt-nanoparticles have been used as active catalysts to initially investigate the viability of MOFs. Also Pt-based molecular catalysts have successfully been incorporated into MOFs either alone or together with photosensitizing units. More recently, Fe- and Co-based molecular catalysts have been employed and we expect this trend to become increasingly popular to create inexpensive, functional materials. As photosensitizers, often well-known molecular dyes such as $\mathrm{Ru}(\mathrm{bpy})_{3} \mathrm{Cl}_{2}$ or less expensive 
porphyrines and Rhodamine B have been used. Derivatives of the two former sensitizers have even been integrated as linker units into MOFs.

While statistic and often unspecific introduction of functional units into MOFs provided a good starting point that led to functional hydrogen evolution photo-catalysts, we expect that future developments in this field will be achieved by more controlled MOF functionalization and a higher control of the positions of the functional units relative to each other. For example, when catalytically active units and photosensitizers are being incorporated into nonconductive frameworks such as UiO-series, the distance between the two actors is crucial for efficient charge transfer and therefore catalysis.

On a different note, catalysis within MOFs may actually not be limited by the intrinsic turnover frequency of the catalyst, but limitations that arise from substrate accessibility. The dimensions of MOF particles often range from several hundred $\mathrm{nm}$ up to $\mu \mathrm{m}$, while individual cavities are about tens of $\AA$ in size, depending on the MOF. Assuming a homogeneous distribution of catalyst throughout the particle, this means that substrates have to diffuse through hundreds of pores to reach catalysts that are deeply buried in the interior of the MOF. It is thus questionable whether all catalytic units are accessible by photo-generated reducing equivalents and protons, or if the process is mainly restricted to near-surface catalysts. While this question has proven a formidable challenge to investigate, it also makes all turnover numbers that are reported in the original papers questionable. In reality, the true turnover numbers and rates may be orders of magnitude higher that reported, as catalysis may be sustained by a small fraction of active catalysts at the surface.

Following this line of thinking, the use of metal-cluster nodes in light-induced linker-to-cluster electron transfer processes may be a promising route to extent the "active portion" of the MOFs. Increasing the amount of active material in this way will probably become more and more important as the utilization of the entire MOF backbone for charge- or energy transfer will help to bridge the distances between catalyst and sensitizer. Also, studying and improving the proton-flux within photo-catalytically active frameworks will become highly important. We believe that method development towards investigation of both charge transfer and substrate movement within the pores will play a very important role in the future to understand the photocatalytic mechanisms in MOFs better and to enhance the efficiencies of these materials.

Acknowledgments S.P. thanks the Kungliga Vetenskapsakademin for a travel scholarship to participate in the ISHHC. This work was further supported by the Swedish Research Council, the Swedish Energy Agency and the Knut \& Alice Wallenberg Foundation.
Open Access This article is distributed under the terms of the Creative Commons Attribution 4.0 International License (http://crea tivecommons.org/licenses/by/4.0/), which permits unrestricted use, distribution, and reproduction in any medium, provided you give appropriate credit to the original author(s) and the source, provide a link to the Creative Commons license, and indicate if changes were made.

\section{References}

1. Eberle U, Felderhoff M, Schüth F (2009) Angew Chem Int Ed 48:6608

2. Schüth F (1984) Chem Ing Tech 2011:83

3. Lewis NS, Nocera DG (2006) Proc Natl Acad Sci 103:15729

4. Magnuson A, Anderlund M, Johansson O, Lindblad P, Lomoth R, Polivka T, Ott S, Stensjö K, Styring S, Sundström V, Hammarström L (1899) Acc Chem Res 2009:42

5. Zou Z, Ye J, Sayama K, Arakawa H (2001) Nature 414:625

6. Suh MP, Park HJ, Prasad TK, Lim D-W (2012) Chem Rev 112:782

7. Sumida K, Rogow DL, Mason JA, McDonald TM, Bloch ED, Herm ZR, Bae T-H, Long JR (2012) Chem Rev 112:724

8. Kreno LE, Leong K, Farha OK, Allendorf M, Van Duyne RP, Hupp JT (2012) Chem Rev 112:1105

9. Della Rocca J, Liu D, Lin W (2011) Acc Chem Res 44:957

10. Horcajada P, Gref R, Baati T, Allan PK, Maurin G, Couvreur P, Férey G, Morris RE, Serre C (2012) Chem Rev 112:1232

11. Lee J, Farha OK, Roberts J, Scheidt KA, Nguyen ST, Hupp JT (2009) Chem Soc Rev 38:1450

12. Ma L, Abney C, Lin W (2009) Chem Soc Rev 38:1248

13. Yoon M, Srirambalaji R, Kim K (2012) Chem Rev 112:1196

14. Meyer K, Ranocchiari M, van Bokhoven JA (1923) Energy Environ Sci 2015:8

15. Wang J-L, Wang C, Lin W (2012) ACS Catal 2:2630

16. Lu W, Wei Z, Gu Z-Y, Liu T-F, Park J, Park J, Tian J, Zhang M, Gentle III T, Bosch M, Zhou H-C, Zhang Q (2014) Chem Soc Rev 43:5561

17. Cohen SM (2012) Chem Rev 112:970

18. Ferey G (2008) Chem Soc Rev 37:191

19. O'Keeffe M, Yaghi OM (2012) Chem Rev 112:675

20. Stock N, Biswas S (2012) Chem Rev 112:933

21. Cavka JH, Jakobsen S, Olsbye U, Guillou N, Lamberti C, Bordiga S, Lillerud KP (2008) J Am Chem Soc 130:13850

22. Valenzano L, Civalleri B, Chavan S, Bordiga S, Nilsen MH, Jakobsen S, Lillerud KP, Lamberti C (2011) Chem Mater 23:1700

23. Ferey G, Latroche M, Serre C, Millange F, Loiseau T, PercheronGuegan A (2003) Chem Commun 24:2976

24. Férey G, Mellot-Draznieks C, Serre C, Millange F, Dutour J, Surblé S, Margiolaki I (2005) Science 309:2040

25. Dan-Hardi M, Serre C, Frot T, Rozes L, Maurin G, Sanchez C, Férey G (2009) J Am Chem Soc 131:10857

26. Feng D, Gu Z-Y, Li J-R, Jiang H-L, Wei Z, Zhou H-C (2012) Angew Chem Int Ed 51:10307

27. Phan A, Doonan CJ, Uribe-Romo FJ, Knobler CB, O'Keeffe M, Yaghi OM (2010) Acc Chem Res 43:58

28. Burtch NC, Jasuja H, Walton KS (2014) Chem Rev 114(10575): 19

29. Katz MJ, Brown ZJ, Colon YJ, Siu PW, Scheidt KA, Snurr RQ, Hupp JT, Farha OK (2013) Chem Commun 49:9449

30. Jasuja H, Jiao Y, Burtch NC, Huang Y-G, Walton KS (2014) Langmuir 30:14300

31. Meilikhov M, Yusenko K, Esken D, Turner S, Van Tendeloo G, Fischer RA (2010) Eur J Inorg Chem 2010:3701 
32. Garibay SJ, Cohen SM (2010) Chem Commun 46:7700

33. Fei H, Cohen SM (2014) Chem Commun 50:4810

34. Fei H, Cohen SM (2015) J Am Chem Soc 137:2191

35. Evans JD, Sumby CJ, Doonan CJ (2014) Chem Soc Rev 43: 5933

36. Deria P, Mondloch JE, Karagiaridi O, Bury W, Hupp JT, Farha OK (2014) Chem Soc Rev 43:5896

37. Fei H, Cahill JF, Prather KA, Cohen SM (2013) Inorg Chem 52:4011

38. Kim M, Cahill JF, Fei H, Prather KA, Cohen SM (2012) J Am Chem Soc 134:18082

39. Kim M, Cahill JF, Su Y, Prather KA, Cohen SM (2012) Chem Sci $3: 126$

40. He J, Wang J, Chen Y, Zhang J, Duan D, Wang Y, Yan Z (2014) Chem Commun 50:7063

41. Wen M, Mori K, Kamegawa T, Yamashita H (2014) Chem Commun 50:11645

42. Yuan Y-P, Yin L-S, Cao S-W, Xu G-S, Li C-H, Xue C (2015) Appl Catal B 168-169:572

43. Pullen S, Fei H, Orthaber A, Cohen SM, Ott S (2013) J Am Chem Soc 135:16997

44. Liu X-L, Wang R, Zhang M-Y, Yuan Y-P, Xue C (2015) APL Mater 3:104403

45. Fei H, Sampson MD, Lee Y, Kubiak CP, Cohen SM (2015) Inorg Chem 54:6821

46. Chambers $\mathrm{MB}$, Wang $\mathrm{X}$, Elgrishi $\mathrm{N}$, Hendon $\mathrm{CH}$, Walsh $\mathrm{A}$, Bonnefoy J, Canivet J, Quadrelli EA, Farrusseng D, MellotDraznieks C, Fontecave M (2015) ChemSusChem 8:603
47. Fateeva A, Chater PA, Ireland CP, Tahir AA, Khimyak YZ, Wiper PV, Darwent JR, Rosseinsky MJ (2012) Angew Chem Int Ed 51:7440

48. Barthelet K, Riou D, Nogues M, Férey G (2003) Inorg Chem 42:1739

49. Hou C-C, Li T-T, Cao S, Chen Y, Fu W-F (2015) J Mater Chem A 3:10386

50. Zhou T, Du Y, Borgna A, Hong J, Wang Y, Han J, Zhang W, Xu R (2013) Energy Environ Sci 6:3229

51. Sasan K, Lin Q, Mao C, Feng P (2014) Chem Commun 50:10390

52. Zhang Z-M, Zhang T, Wang C, Lin Z, Long L-S, Lin W (2015) J Am Chem Soc 137:3197

53. Gomes Silva C, Luz I, Llabrés i Xamena FX, Corma A, García H (2010) Chem-A European J 16:11133

54. Horiuchi Y, Toyao T, Saito M, Mochizuki K, Iwata M, Higashimura H, Anpo M, Matsuoka M (2012) J Phy Chem C 116:20848

55. Nasalevich MA, Becker R, Ramos-Fernandez EV, Castellanos S, Veber SL, Fedin MV, Kapteijn F, Reek JNH, van der Vlugt JI, Gascon J (2015) Energy Environ Sci 8:364

56. Lin R, Shen L, Ren Z, Wu W, Tan Y, Fu H, Zhang J, Wu L (2014) Chem Commun 50:8533

57. He J, Yan Z, Wang J, Xie J, Jiang L, Shi Y, Yuan F, Yu F, Sun Y (2013) Chem Commun 49:6761

58. Zhou J-J, Wang R, Liu X-L, Peng F-M, Li C-H, Teng F, Yuan Y-P (2015) Appl Surf Sci 346:278

59. Wang R, Gu L, Zhou J, Liu X, Teng F, Li C, Shen Y, Yuan Y (2015) Adv Mater Inter 2:1500037 